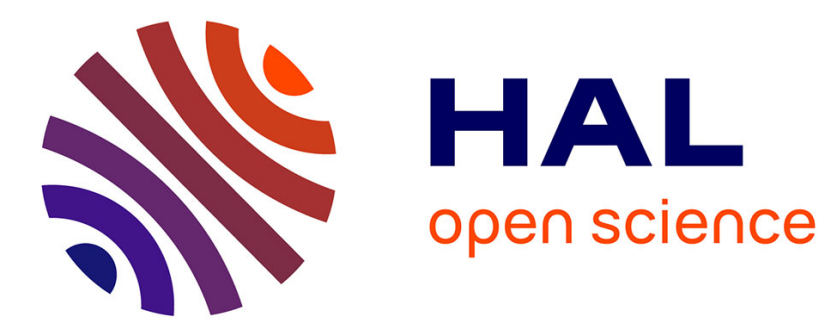

\title{
From random walks to spin glasses
}

Bernard Derrida

\section{To cite this version:}

Bernard Derrida. From random walks to spin glasses. Physica D: Nonlinear Phenomena, 1997, 16th Annual International Conference of the Center for Nonlinear Studies, 107 (2-4), pp.186-198. 10.1016/S0167-2789(97)00086-9 . hal-03282984

\section{HAL Id: hal-03282984 https://hal.science/hal-03282984}

Submitted on 19 Jul 2021

HAL is a multi-disciplinary open access archive for the deposit and dissemination of scientific research documents, whether they are published or not. The documents may come from teaching and research institutions in France or abroad, or from public or private research centers.
L'archive ouverte pluridisciplinaire HAL, est destinée au dépôt et à la diffusion de documents scientifiques de niveau recherche, publiés ou non, émanant des établissements d'enseignement et de recherche français ou étrangers, des laboratoires publics ou privés. 


\title{
From random walks to spin glasses
}

\author{
B. Derrida ${ }^{1}$ \\ Laboratoire de Physique Statistique, E.N.S. 24 rue Lhomond, F-75005 Paris, France
}

\begin{abstract}
The talk was a short review on systems which exhibit non-self-averaging effects: sums of random variables when the distribution has a long tail, mean field spin glasses, random map models and returns of a random walk to the origin. Non-selfaveraging effects are identical in the case of sums of random variables and in the spin glass problem as predicted by the replica approach. Also we will see that for the random map models or for the problem of the returns of a random walk to the origin, the non-self-averaging effects coincide with the results of the replica approach when the number $n$ of replica $n=-1 / 2$ or $n=-1$.
\end{abstract}

Keywords: Spin glass; Broken symmetry of replica; Random walks; Non-self-averaging effects; Levy distributions

\section{Introduction}

An important outcome $[1,2]$ of the replica theory of spin glasses has been the prediction of non-self-averaging effects: in the low temperature phase, phase space can be thought of as if it was decomposed into infinitely many pure states $\alpha$, the weights of which remain sample dependent even in the thermodynamic limit (i.e. when the system size becomes infinite). The fluctuations of these weights $W_{\alpha}$ can be described by considering their moments $Y_{k}$ defined by

$$
Y_{k}=\sum_{\alpha}\left(W_{\alpha}\right)^{k}
$$

where in (1) the sum runs over all the pure states $\alpha$. The fact that these moments $Y_{k}$ have non-trivial probability distributions, even in the thermodynamic limit appears as a signature of the presence of non-self-averaging effects in the spin glass phase.

The goal of this lecture is to show that similar non-self-averaging effects are present in much simpler systems such as sums of identically distributed random variables or random map models. We will see in particular that the statistical properties of the moments $Y_{k}$ are identical in the case of sums of random variables and of mean field spin glasses. We will also see that the statistical properties of the moments $Y_{k}$ for several other systems (random map models, returns of a random walk to its starting point) coincide with what one would obtain from the Parisi scheme

\footnotetext{
${ }^{1}$ E-mail: bernard.derrida@physique.ens.fr.
} 
for breaking the symmetry between the replica for some unusual limits of the number $n$ of replica $(n=-1 / 2$ or $n=-1$ ).

The presentation of this lecture is as follows: the case of sums of positive random variables is discussed in Section 2. The replica approach for mean field spin glasses is presented in Section 3 and the calculation of the $Y_{k}$ within the replica scheme is given in Section 4. Section 5 shows how the $Y_{k}$ can be computed for random map models whereas the problem of the returns of a random walk to its starting point is discussed in Section 6 . After the conclusion in Section 7, some recent developments are discussed in Section 8.

\section{Sums of random variables}

Let us consider the sum $S_{N}$ of $N$ independent random variables $x_{1}, \ldots, x_{N}$

$$
S_{N}=\sum_{\alpha=1}^{N} x_{\alpha}
$$

We assume that these variables are all positive and that they are distributed according to a probability distribution $\rho(x)$ which decays slowly as $x \rightarrow \infty$

$$
\rho(x) \simeq \frac{A}{x^{1+\mu}}
$$

with $0<\mu<1$. For large $N$, it is well known that $S_{N} / N^{1 / \mu}$ has a stable distribution called a Levy distribution (see [3] and references therein). One can define the weight $W_{\alpha}$ of the term $x_{\alpha}$ in the sum as

$$
W_{\alpha}=\frac{x_{\alpha}}{S_{N}} .
$$

Obviously one has

$$
Y_{1}=\sum_{\alpha} W_{\alpha}=1
$$

For large $N$, one can show that the moments $Y_{k}$ defined by (1) (for $k>\mu$ ) have a probability distribution which depends only on $\mu$ and $k$ (and becomes independent of $N$ or $A$ ). For example, one can calculate $\left\langle Y_{k}\right\rangle$ in the large $N$ limit by using the following identity

$$
Y_{k}=\sum_{\alpha} \frac{x_{\alpha}^{k}}{\left(\sum_{\beta} x_{\beta}\right)^{k}}=\int_{0}^{\infty} \frac{t^{k-1} \mathrm{~d} t \mathrm{e}^{-t \sum_{\beta} x_{\beta}}}{\Gamma(k)} \sum_{\alpha} x_{\alpha}^{k} .
$$

(This is a direct consequence of the definition of the $\Gamma$ function: $\Gamma(z)=\int_{0}^{\infty} t^{z-1} \mathrm{e}^{-t} \mathrm{~d} t$.) When one averages over the $x_{\beta}$, one gets

$$
\left\langle Y_{k}\right\rangle=\frac{N}{\Gamma(k)} \int_{0}^{\infty} t^{k-1} \mathrm{~d} t\left\langle\mathrm{e}^{-t x}\right\rangle^{N-1}\left\langle x^{k} \mathrm{e}^{-t x}\right\rangle
$$

For large $N$, the integral is dominated by the small $t$ behavior. One can easily see that for small $t$

$$
\left\langle\mathrm{e}^{-t x}\right\rangle=1-\int_{0}^{\infty}\left(1-\mathrm{e}^{-t x}\right) \rho(x) \mathrm{d} x \simeq \exp \left[-t^{\mu} A(-\Gamma(-\mu))\right]
$$


whereas for $k>\mu$, one has

$$
\left\langle x^{k} \mathrm{e}^{-t x}\right\rangle=\int_{0}^{\infty} x^{k} \mathrm{e}^{-t x} \rho(x) \mathrm{d} x \simeq A t^{\mu-k} \Gamma(k-\mu) .
$$

This gives for large $N$

$$
\left\langle Y_{k}\right\rangle \simeq \frac{N A \Gamma(k-\mu)}{\Gamma(k)} \int_{0}^{\infty} t^{\mu-1} \mathrm{~d} t \exp \left[-(N-1) t^{\mu} A(-\Gamma(-\mu))\right],
$$

which for $N \rightarrow \infty$ leads to

$$
\left\langle Y_{k}\right\rangle_{\text {Levy }}=\frac{\Gamma(k-\mu)}{\Gamma(k) \Gamma(1-\mu)} .
$$

Exact expressions of all the correlation functions between the $Y_{k}$ can be obtained by similar (but of course longer) calculations. For example, for $k>\mu$ and $k^{\prime}>\mu$, one can show that

$$
\left\langle Y_{k} Y_{k^{\prime}}\right\rangle_{\text {Levy }}=\frac{\Gamma\left(k+k^{\prime}-\mu\right)}{\Gamma(1-\mu) \Gamma\left(k+k^{\prime}\right)}+\mu \frac{\Gamma(k-\mu) \Gamma\left(k^{\prime}-\mu\right)}{[\Gamma(1-\mu)]^{2} \Gamma\left(k+k^{\prime}\right)} .
$$

Remark 1 . The fact that $\left\langle Y_{k}^{2}\right\rangle \neq\left\langle Y_{k}\right\rangle^{2}$ indicates that the distribution of $Y_{k}$ remains broad even in the large $N$ limit. Therefore, the moments $Y_{k}$ and consequently the weights $W_{\alpha}$ are non-self-averaging quantities.

Remark 2. A consequence of the fact that $\left\langle Y_{k}\right\rangle$ has a non-zero limit as $N \rightarrow \infty$ is that the largest term in the sum $S_{N}$ contributes as a non-zero fraction to the sum $S_{N}$. Indeed, if one defines $W_{\max }$ by

$$
W_{\max }=\max _{\alpha} W_{\alpha},
$$

one has

$$
\left\langle Y_{k}\right\rangle=\left\langle\sum_{\alpha} W_{\alpha}^{k}\right\rangle \leq\left\langle\left(W_{\max }\right)^{k-1}\right\rangle .
$$

So the fact (11) that $\left\langle Y_{k}\right\rangle>0$ implies that $W_{\max }>0$ with at least a non-zero probability.

Remark 3. There exists an easy way of generating by a Monte Carlo procedure the probability distributions of the $Y_{k}$. To do so one can generate an infinite sequence of independent random numbers $z_{1}, z_{2}, \ldots$, uniformly distributed between 0 and 1 and one can contruct a sequence $\omega_{1}, \omega_{2}, \ldots$, by

$$
\begin{aligned}
\omega_{1} & =\left(-\log z_{1}\right)^{-1 / \mu}, \\
\omega_{i+1} & =\omega_{i}\left(1-\omega_{i}^{\mu} \log z_{i+1}\right)^{-1 / \mu} .
\end{aligned}
$$

Then the $Y_{k}$ computed by

$$
Y_{k}=\sum_{i=1}^{\infty}\left(\frac{\omega_{i}}{\sum_{n \geq 1} \omega_{n}}\right)^{k}
$$

has the same distribution as the $Y_{k}$ defined by (1) and (4). In fact, one can show that $\omega_{1} / \sum_{n} \omega_{n}$ has the same distribution as $W_{\max }, \omega_{2} / \sum_{n} \omega_{n}$ has the same distribution as the second largest weight and so on. So a good approximation to the $Y_{k}$ is to keep only the first few terms in the sums over $i$ and $n$ which appear in (17). 


\section{Mean field spin glasses}

We are going to see now that quantities similar to the $Y_{k}$ can be introduced also in the spin glass problem. To do that one needs to recall briefly the Parisi replica approach $[1,2,4]$ to the Sherrington-Kirkpatrick model $[5,6]$.

The Sherrington-Kirkpatrick model is a model of $N$ Ising spins $S_{i}= \pm 1$ which interact with random long-range interactions. The system has $2^{N}$ possible spin configurations and the energy $E_{\alpha}$ of a configuration $\alpha \equiv\left\{S_{i}^{\alpha}\right\}$ is given by

$$
E_{\alpha}=-\sum_{1 \leq i<j \leq N} J_{i j} S_{i}^{\alpha} S_{j}^{\alpha}
$$

In the Sherrington-Kirkpatrick model, for each pair $i j$ of spins, there is a random interaction $J_{i j}$ chosen according to

$$
\rho\left(J_{i j}\right)=\sqrt{\frac{N-1}{2 \pi}} \exp \left(-\frac{(N-1) J_{i j}^{2}}{2}\right) .
$$

A given sample corresponds to a random choice of the interactions $\left\{J_{i j}\right\}$ and the partition function of each sample is given by

$$
Z\left(\left\{J_{i j}\right\}\right)=\sum_{\alpha=1}^{2^{N}} \exp \left(-\frac{E_{\alpha}}{T}\right),
$$

where $T$ is the temperature. As usual in the theory of disordered systems, the meaningful quantity to consider is $\langle\log Z\rangle$, the average of $\log Z$ over all the possible realizations of the interactions $J_{i j}$.

A possible approach to try to calculate $\langle\log Z\rangle$ is to use the replica method. So far, the replica approach is still mathematically poorly understood, but it is very much used in the theory of disordered systems, mostly because in many cases, it is the only available approach. There are, in fact, only a few problems like the random energy models or the mean field theory of directed polymers in a random medium for which there exist alternative approaches to compute $\langle\log Z\rangle$ and for which the replica approach, with a symmetry broken as predicted by the Parisi approach, has been shown to give the right answer [7-9].

The replica method to calculate $(\log Z)$ consists of two steps. First, one computes the integer moments $\left\langle Z^{n}\right\rangle$ of the partition function $Z$ for all integer $n$. Then, one tries to use the following limiting procedure:

$$
\langle\log Z\rangle=\lim _{n \rightarrow 0} \frac{\log \left(Z^{n}\right)}{n} .
$$

In general, it is the second step which is mathematically hard to justify, mostly because the knowledge of $\left\langle Z^{n}\right\rangle$ for integer $n$ is usually not sufficient to determine the value of that quantity for non-integer $n$.

Let us see how the first step can be done. The calculation of the first moment of $Z$ is trivial and one finds

$$
\langle Z\rangle=2^{N} \exp \frac{N}{4 T^{2}} .
$$

The calculation of the other integer moments of $Z$ requires a little more work. For example the second moment $\left\langle Z^{2}\right\rangle$ is given by

$$
\left\langle Z^{2}\right\rangle=\sum_{\alpha=1}^{2^{N}} \sum_{\beta=1}^{2^{N}}\left\langle\exp \left(-\frac{E_{\alpha}+E_{\beta}}{T}\right)\right\rangle .
$$


It can be seen easily from expressions (18) and (19) that $\left\langle\exp -\left(E_{\alpha}+E_{\beta}\right) / T\right\rangle$ depends only on the overlap $q_{\alpha \beta}$ between the two configurations $\alpha$ and $\beta$

$$
q_{\alpha \beta}=\frac{1}{N} \sum_{i=1}^{N} S_{i}^{\alpha} S_{i}^{\beta} .
$$

For large $N$ it can be written as

$$
\left\langle\exp \left(-\frac{E_{\alpha}+E_{\beta}}{T}\right)\right\rangle \sim \exp \left[N f\left(q_{\alpha \beta}\right)\right]
$$

with the function $f(q)=\left(1+q^{2}\right) / T^{2}$. Thus to calculate the large $N$ behavior of $\left\langle Z^{2}\right\rangle$, it is sufficient to know the number of pairs of configurations $\alpha$ and $\beta$ having a given overlap $q_{\alpha \beta}$ and to sum over $q_{\alpha \beta}$. The number of pairs of configurations having a given overlap $q_{\alpha \beta}$ is an entropic term which behaves like $\exp \left[N s\left(q_{\alpha \beta}\right)\right]$ for large $N$. Therefore, the large $N$ behavior of $\left\langle Z^{2}\right\rangle$ can be determined by a saddle point method

$$
\frac{\log \left\langle Z^{2}\right\rangle}{N}=\max _{q_{\alpha \beta}}\left\{f\left(q_{\alpha \beta}\right)+s\left(q_{\alpha \beta}\right)\right\}
$$

This reasoning can be easily extended to calculate all higher integer moments and for each $n$, the large $N$ behavior of $\left\langle Z^{n}\right\rangle$ can be obtained as a saddle point in a space of the $n(n-1) / 2$ variables $q_{\alpha \beta}$ :

$$
\frac{\log \left\langle Z^{n}\right\rangle}{N}=\max _{\left\{q_{\alpha \beta}\right\}} g\left(\left\{q_{\alpha \beta}\right\}, n\right),
$$

where the function $g\left(\left\{q_{\alpha \beta}\right\}, n\right)$ contains both the energy and the entropy terms.

\section{The Parisi broken symmetry of replica}

Even when $n$ is an integer, finding the maximum of a complicated function of $n(n-1) / 2$ parameters is a difficult task. It turns out that for integer $n$, the maximum is symmetric $[10,11]$, i.e. all the $q_{\alpha \beta}$ are equal to some value $q$. Then the problem of finding the maximum is greatly simplified as one needs to find the maximum of a function of a single variable $q$. This is what Sherrington and Kirpatrick [5,6] originally did to calculate the moments $\left\langle Z^{n}\right\rangle$. The $n$ dependence was simple enough that they could obtain an expression of $\langle\log Z\rangle$ via the formula (21). However, they noticed from the very beginning that their expression could not be correct as it predicted a negative entropy at sufficiently low temperature.

Several works followed the one of Sherrington-Kirkpatrick to test the assumption that the saddle point was symmetric [12,13]. In 1979, Parisi proposed an ansatz for which this symmetry was broken $[2,4]$. His idea was to assume that the $q_{\alpha \beta}$ depends on the pair $\alpha \beta$ in such a way that the calculation can be done for any value of $n$, the number of replica. In its simplest version, one groups the $n$ replica into $n / \mu$ blocks of $\mu$ replica each. One takes two possible values $q_{1}$ and $q_{2}$ for the overlap and one looks for a saddle point such that $q_{\alpha \beta}=q_{1}$ whenever $\alpha$ and $\beta$ belong to the same group and $q_{\alpha \beta}=q_{2}$ when $\alpha$ and $\beta$ belong to different groups. For this saddle point the function $g\left(\left\{q_{\alpha \beta}\right\}, n\right)$ becomes a function of $q_{1}, q_{2}$ and $\mu$ and one tries to find the extremum with respect to these three parameters $q_{1}, q_{2}$ and $\mu$. (In fact what Parisi proposed was a little more complicated as he considered situations where the blocks were themselves decomposed into blocks and so on.)

Of course, this kind of saddle point is in principle acceptable only if $n$ is an integer and if it is divisible by $\mu$. However, Parisi proposed to forget the fact that $n$ was an integer, and he suggested that all the calculations be done as 
if $n, n / \mu$ and $\mu$ were integers and at the end of the calculation the limit $n \rightarrow 0$ be taken, by replacing the constraint that $1 \leq \mu \leq n$, by $0 \leq \mu<1$. I shall not try to explain why this procedure is believed to lead to the correct expression of $\langle\log Z\rangle[1,2,4,14]$. Here, I just want to show that within this replica approach the calculation of the moments of the $Y_{k}$ becomes extremely easy.

Consider a system of $n$ replica (or $n$ objects) grouped into $n / \mu$ blocks of $\mu$ replica each. If one chooses $k$ different replica among these $n$ replica, the probability that the $k$ replica are in the same group is given by

$$
\left\langle Y_{k}\right\rangle_{\text {replica }}=\frac{n(\mu-1)(\mu-2) \cdots(\mu-k+1)}{n(n-1)(n-2) \cdots(n-k+1)} .
$$

For non-integer $n$ this can be written as

$$
\left\langle Y_{k}\right\rangle_{\text {replica }}=\frac{\Gamma(k-\mu)}{\Gamma(1-\mu)} \frac{\Gamma(1-n)}{\Gamma(k-n)}
$$

with the convention that when $n$ is an integer, one takes the limit of this expression as $n$ tends to its integer value.

Now let us assume that for a given choice of the interactions $J_{i j}$, phase space is decomposed into regions of weights $W_{\alpha}$. If one considers $k$ realizations of the same sample (i.e. $k$ copies of the system with the same set of interactions, or $k$ 'typical' spin configurations of the same sample), the probability that the $k$ of them are in the same region of phase space is

$$
Y_{k}=\sum_{\alpha}\left(W_{\alpha}\right)^{k}
$$

When one tries to compute this expression within the replica approach [1,2], each realization appears as a different replica and on averaging over the disorder, one ends up with expression (29) in the limit $n \rightarrow 0$. Therefore, for the mean field spin glass problem, the Parisi approach predicts

$$
\left\langle Y_{k}\right\rangle_{\text {spinglass }}=\lim _{n \rightarrow 0}\left\langle Y_{k}\right\rangle_{\text {replica }}=\frac{\Gamma(k-\mu)}{\Gamma(1-\mu) \Gamma(k)}
$$

and the value of $\mu$ which enters in this expression is a complicated function of the temperature $T$ as it is the optimal value of $\mu$ when one tries to find the extremum of the function $g\left(\left\{q_{\alpha \beta}\right\}, n\right)$.

One can repeat this calculation for all the correlation functions between the $Y_{k}$ : for example, if we have $n$ replica grouped into $n / \mu$ blocks of $\mu$ replica each, the probability that $k$ of them are together in a block and $k^{\prime}$ of them are also together in a block is given by

$$
\begin{aligned}
& \frac{n(\mu-1)(\mu-2) \cdots\left(\mu-k-k^{\prime}+1\right)}{n(n-1)(n-2) \cdots\left(n-k-k^{\prime}+1\right)} \\
& \quad+\frac{n(\mu-1)(\mu-2) \cdots(\mu-k+1)(n-\mu)(\mu-1)(\mu-2) \cdots\left(\mu-k^{\prime}+1\right)}{n(n-1)(n-2) \cdots\left(n-k-k^{\prime}+1\right)} .
\end{aligned}
$$

The first term represents situations where the $k+k^{\prime}$ replica are all in the same block whereas the second term represents the situations where the first $k$ replica are in one block and the other $k^{\prime}$ replica are in a different block. As before, one can rewrite (32) as

$$
\left\langle Y_{k} Y_{k^{\prime}}\right\rangle_{\text {replica }}=\frac{\Gamma\left(k+k^{\prime}-\mu\right) \Gamma(1-n)}{\Gamma(1-\mu) \Gamma\left(k+k^{\prime}-n\right)}+(\mu-n) \frac{\Gamma(k-\mu) \Gamma\left(k^{\prime}-\mu\right) \Gamma(1-n)}{[\Gamma(1-\mu)]^{2} \Gamma\left(k+k^{\prime}-n\right)} .
$$

Then the correlation function $\left\langle Y_{k} Y_{k^{\prime}}\right\rangle$ for the spin glass problem is given by

$$
\left\langle Y_{k} Y_{k^{\prime}}\right\rangle_{\text {spinglass }}=\lim _{n \rightarrow 0}\left\langle Y_{k} Y_{k^{\prime}}\right\rangle_{\text {replica }}=\frac{\Gamma\left(k+k^{\prime}-\mu\right)}{\Gamma(1-\mu) \Gamma\left(k+k^{\prime}\right)}+\mu \frac{\Gamma(k-\mu) \Gamma\left(k^{\prime}-\mu\right)}{[\Gamma(1-\mu)]^{2} \Gamma\left(k+k^{\prime}\right)} .
$$


Remark 4. We see from (31), (34) that the results are identical to expressions (11), (12) of Section 2, obtained for sums of random variables. Therefore, the statistical properties of the weights of the pure states of spin glasses as given by the replica theory are the same as what one obtains in the case of sums of random variables with long tails.

Remark 5. The simplest way of understanding the relation between spin glasses and sums of random variables is probably to consider the random energy model $[7,15,16]$, for which the calculation of the moments $Y_{k}$ can be done either by the replica method or by an approach very similar to the one presented in Section 2 . This was first pointed out to me by Jean-Philippe Bouchaud who noticed that the non-integer moments of the partition function of the random energy model [17] have identical expressions as the moments of random variables distributed according to Levy distributions.

Remark 6. We will see in Sections 5 and 6 that expressions (29) and (33) give also the moments of the $Y_{k}$ when one chooses for $n$ values other than $n=0$.

\section{Random map models}

We are now going to see that the moments and the correlations of the $Y_{k}$ have very similar expressions in several other examples. We will discuss in this section the case of random map models, which appear as very simplified mathematical problems related to the theory of random networks of automata such as the Kauffman model $[18,19]$ and in Section 6 the problem of the returns to the origin of a random walk.

I shall consider here two cases: first, the random map model [20] without any constraint, and then the area preserving random map model.

\subsection{The random map model}

One considers a system which consists of $M$ possible configurations. The dynamics is determined by a random map $F$ in this phase space: if the system is in configuration $C_{t}$ at time $t$, then its configuration $C_{t+1}$ at time $t+1$ is given by

$$
C_{t+1}=F\left(C_{t}\right) .
$$

By definition of the model the function $F$ is totally random, i.e. for each $C, F(C)$ is chosen at random among the $M$ configurations with equal probability. As the map is deterministic and phase space is finite, all the trajectories converge to periodic orbits (or fixed points). Thus phase space can be decomposed into the basins of attraction of the different periodic orbits.

One can define the weight $W_{\alpha}$ of a given orbit $\alpha$ as the fraction of phase space belonging to its basin. This means that

$$
W_{\alpha}=\frac{\Omega_{\alpha}}{M}
$$

where $\Omega_{\alpha}$ is the number of points in the basin of the attractor $\alpha$. Once the weights $W_{\alpha}$ are defined, one can consider their moments $Y_{k}$ with the usual relation (1)

$$
Y_{k}=\sum_{\alpha}\left(W_{\alpha}\right)^{k}
$$

From this expression, it is easy to see that $Y_{k}$ is the probability that $k$ randomly chosen configurations belong to the same basin. If we choose $k$ random initial configurations $C^{1}, C^{2}, \ldots, C^{k}$ converging to the same attractor and if we 
call $m_{1}$ the number of different configurations in the trajectory of $C^{1}, m_{2}$, the number of different configurations in the union of the trajectories of $C^{1}$ and $C^{2}, \ldots, m_{\lambda}$, the number of different configurations in the union of the trajectories of $C^{1}, \ldots, C^{\lambda}$, one has [20]

$$
\left\langle Y_{k}\right\rangle=\sum_{m_{k}=1}^{\infty} \sum_{m_{k-1}=1}^{m_{k}} \cdots \sum_{m_{1}=1}^{m_{2}}\left\{\prod_{i=0}^{m_{k}-1}\left(1-\frac{i}{M}\right)\right\} m_{1} \frac{m_{1} m_{2} m_{3} \cdots m_{k-1}}{M^{k}}
$$

For large $M$, the sums become integrals and if one makes the change of variables $m_{\lambda}=\sqrt{M} x_{\lambda}$, one gets

$$
\left\langle Y_{k}\right\rangle=\int_{0}^{\infty} \mathrm{d} x_{k} \int_{0}^{x_{k}} \mathrm{~d} x_{k-1} \cdots \int_{0}^{x_{2}} \mathrm{~d} x_{1} x_{1}^{2} x_{2} x_{3} \cdots x_{k-1} \mathrm{e}^{-x_{k}^{2} / 2} .
$$

One can perform these integrals and one gets

$$
\left\langle Y_{k}\right\rangle_{\text {random map }}=\frac{\Gamma(k) \Gamma(3 / 2)}{\Gamma(k+1 / 2)} .
$$

The calculation of the correlations between the $Y_{k}$ can be done in a very similar way. For example, one can use the fact that

$$
\left\langle Y_{k} Y_{k^{\prime}}\right\rangle=\sum_{\alpha}\left(W_{\alpha}\right)^{k+k^{\prime}}+\sum_{\alpha \neq \beta}\left(W_{\alpha}\right)^{k}\left(W_{\beta}\right)^{k^{\prime}}
$$

The first term corresponds to $k+k^{\prime}$ configurations belonging to the same basin and the second term to the first $k$ configurations belonging to one basin and the other $k^{\prime}$ configurations belonging to a different basin. The calculation follows the same steps as before and one obtains

$$
\left\langle Y_{k} Y_{k^{\prime}}\right\rangle_{\mathrm{random} \text { map }}=\frac{\Gamma\left(k+k^{\prime}\right) \Gamma(3 / 2)}{\Gamma\left(k+k^{\prime}+1 / 2\right)}+\frac{1}{2} \frac{\Gamma(k) \Gamma\left(k^{\prime}\right) \Gamma(3 / 2)}{\Gamma\left(k+k^{\prime}+1 / 2\right)} .
$$

Remark 7. We see from (40) and (42) that in general $\left\langle Y_{k}^{2}\right\rangle \neq\left\langle Y_{k}\right\rangle^{2}$. Thus for the random map model too, the moments $Y_{k}$ and the weights $W_{\alpha}$ are non-self-averaging quantities (for example $\left\langle Y_{2}\right\rangle=2 / 3$ whereas $\left\langle Y_{2}^{2}\right\rangle=52 / 105 \neq\left\langle Y_{2}\right\rangle^{2}$ ).

Remark 8. It is interesting to notice as was first pointed out to me by Giorgio Parisi that expressions (40) and (42) are exactly the same as those obtained in (29) and (33) in the limit $\mu=0$ and $n=-1 / 2$. So the non-self-averaging effects are exactly the same for the random map model as what one gets from the replica calculation in the case of $-1 / 2$ replica blocked into infinitely many blocks of 0 replica each. The reason for this identity between the results obtained in the random map model and the replica approach for this special choice of $n$ and $\mu$ is so far a coincidence. Of course, it would be interesting to try to develop a replica approach to random map models in order to see whether these values of $n$ and $\mu$ come out in a natural way.

\subsection{The area preserving random map model}

A simple extension of the previous model is the area preserving random map model. In that case, the map $F$ is random as before except that it is a one-to-one map. So $F$ is a random permutation of the $M$ points of phase space. In that case, obviously the basin of attraction of an attractor and the attractor itself coincide. So each point of phase 
space belongs to an attractor and the period of the attractor $\alpha$ is equal to the number of points $\Omega_{\alpha}$ of this attractor. Given a point chosen at random in phase space, the probability $P(\Omega)$ that it belongs to an attractor of $\Omega$ points is

$$
P(\Omega)=\frac{1}{M !} \frac{(M-1) !}{(M-\Omega) !(\Omega-1) !}(M-\Omega) !(\Omega-1) ! .
$$

Each term in this product can be understood easily: the first term corresponds to the total number of functions $F$; the second to choosing the $\Omega-1$ other points of the attractor among the $M-1$ other points of phase space; the third term is the number of permutations of the remaining $M-\Omega$ points of phase space; the fourth term corresponds to the number of ways that the $\Omega-1$ other points of the attractor are visited by the dynamics. This expression is easy to simplify to give

$$
P(\Omega)=\frac{1}{M}
$$

Similarly, it is easy to show that the probability $Q\left(\Omega, \Omega^{\prime}\right)$ that two different points belong to two different attractors of periods $\Omega$ and $\Omega^{\prime}$ is given by

$$
Q\left(\Omega, \Omega^{\prime}\right)=\frac{1}{M(M-1)} .
$$

Then one can deduce from that the expressions of $\left\langle Y_{k}\right\rangle$ and $\left\langle Y_{k} Y_{k^{\prime}}\right\rangle$ by

$$
\left\langle Y_{k}\right\rangle=\sum_{\Omega=1}^{M} P(\Omega) \Omega^{k-1}
$$

and

$$
\left\langle Y_{k} Y_{k^{\prime}}\right\rangle=\sum_{\Omega=1}^{M} P(\Omega) \Omega^{k+k^{\prime}-1}+\sum_{\Omega=1}^{M-1} \sum_{\Omega^{\prime}=1}^{M-\Omega} Q\left(\Omega, \Omega^{\prime}\right) \Omega^{k-1} \Omega^{\prime k^{\prime}-1}
$$

For large $M$, this leads to the following expressions:

$$
\begin{aligned}
& \left\langle Y_{k}\right\rangle_{\text {area preserving }}=\frac{1}{k}, \\
& \left\langle Y_{k} Y_{k^{\prime}}\right\rangle_{\text {area preserving }}=\frac{1}{k+k^{\prime}}+\frac{\Gamma(k) \Gamma\left(k^{\prime}\right)}{\Gamma\left(k+k^{\prime}+1\right)} .
\end{aligned}
$$

Remark 9. We see from (48) and (49) that $\left\langle Y_{k}^{2}\right\rangle \neq\left\langle Y_{k}\right\rangle^{2}$, and thus here again, the moments $Y_{k}$ and the weights $W_{\alpha}$ are non-self-averaging quantities (for example $\left\langle Y_{2}\right\rangle=1 / 2$ whereas $\left\langle Y_{2}^{2}\right\rangle=7 / 24 \neq\left\langle Y_{2}\right\rangle^{2}$ ).

Remark 10. In this case too, expressions (48) and (49) are identical to what the replica calculation (29) and (33) would give for $n=-1$ and $\mu=0$.

\section{On the returns to the origin of a random walk}

Let us finally discuss a very simple problem related to the statistics of the returns of a one-dimensional random walk to the origin. Consider a random walk of $2 T$ steps starting and ending at the origin. Each step is either +1 or -1 with equal probability. There are of course

$$
Z_{T}=\frac{(2 T) !}{T ! T !}
$$


such walks. For each walk, one can decompose the interval $2 T$ into subintervals $2 t_{1}, 2 t_{2}, \cdots 2 t_{i} \cdots$, where $2 t_{i}$ is the time interval separating the $(i-1)$ th and the $i$ th visits of the origin by the walk. Of course, one has

$$
T=t_{1}+t_{2} \cdots+t_{i} \cdots
$$

So the interval of time $2 T$ is partitioned into subintervals delimited by the successive returns to the origin. For each walk, one can define the weight $W_{i}$ of the $i$ th subinterval as

$$
W_{i}=\frac{t_{i}}{T}
$$

and the $Y_{k}$ as

$$
Y_{k}=\sum_{i}\left(W_{i}\right)^{k}
$$

Thus if one chooses $k$ times at random between 0 and $2 T,\left\langle Y_{k}\right\rangle$ is the probability that these $k$ times fall in the same subinterval (this is because, the probability for one time to fall in the $i$ th subinterval is $W_{i}$, and for $k$ times to fall in the $i$ th subintervals this probability is $W_{i}^{k}$ ). In the large $T$ limit, one can obtain the following exact expressions:

$$
\begin{aligned}
& \left\langle Y_{k}\right\rangle_{\text {random walk }}=\frac{1}{2 k-1}, \\
& \left\langle Y_{k} Y_{k^{\prime}}\right\rangle_{\text {random walk }}=\frac{1}{2\left(k+k^{\prime}\right)-1}+\frac{\Gamma(k-1 / 2) \Gamma\left(k^{\prime}-1 / 2\right)}{2 \Gamma(1 / 2) \Gamma\left(k+k^{\prime}+1 / 2\right)},
\end{aligned}
$$

where $\left(>\right.$ denotes the average over the $Z_{T}$ walks. The derivation of (54) and (55) is not difficult. One needs the expression of the number $Q_{T}$ of walks starting at the origin and returning to the origin for the first time after $2 T$ steps.

$$
Q_{T}=2 \frac{(2 T-2) !}{T !(T-1) !} .
$$

One can show that

$$
\left\langle Y_{k}\right\rangle=\frac{1}{Z_{T}} \sum_{t=0}^{T} \sum_{\tau=0}^{T-t} Z_{\tau} Q_{t} Z_{T-t-\tau}\left(\frac{t}{T}\right)^{k}
$$

The generic term in this sum represents a subinterval of length $2 t$ starting at time $2 \tau$ and it gives to the probability over all walks that this subinterval contibutes to (53). Using the asymptotic forms of $Z_{T}$ and $Q_{T}$ for large $T$

$$
Z_{T} \simeq \frac{2^{2 T}}{\sqrt{\pi T}} \text { and } Q_{T} \simeq \frac{2^{2 T}}{2 \sqrt{\pi T^{3}}},
$$

one obtains for large $T$ expression (54).

Similarly, $\left\langle Y_{k} Y_{k^{\prime}}\right\rangle$ is the probability that if one chooses $k+k^{\prime}$ times at random, the first $k$ of them fall in the same subinterval and the last $k^{\prime}$ of them fall in another (possibly the same) subinterval. If one distiguishes the two cases, i.e. the case where the two subintervals are identical and the case where they are different, one finds

$$
\left\langle Y_{k} Y_{k^{\prime}}\right\rangle=\left\langle Y_{k+k^{\prime}}\right\rangle+\frac{1}{Z_{T}} \sum_{t_{1}=0}^{T} \sum_{t_{2}=0}^{T-t_{1}} \sum_{\tau_{1}=0}^{T-t_{1}-t_{2}} \sum_{\tau_{2}=0}^{T-t_{1}-t_{2}-\tau_{1}} Z_{\tau_{1}} Q_{t_{1}} Z_{\tau_{2}} Q_{t_{2}} Z_{T-t_{1}-t_{2}-\tau_{1}-\tau_{2}} \frac{t_{1}^{k} t_{2}^{k^{\prime}}+t_{1}^{k^{\prime}} t_{2}^{k}}{T^{k+k^{\prime}}},
$$

which again leads to (55) in the large $T$ limit. 
Remark 11. Expressions (54) and (55) correspond again to a special case of (29) and (33) for $n=-1 / 2$ and $\mu=1 / 2$. Therefore this problem corresponds to broken replica symmetry for which $-1 / 2$ replica are grouped into -1 block of $1 / 2$ replica each.

\section{Conclusion}

This lecture was an attempt to show that very similar non-self-averaging effects occur in a variety of systems. We saw in particular that the prediction of the Parisi replica theory for spin glasses (31), (34) gives exactly the same answer as a much simpler problem, that of sums of random variables (11), (12). Moreover, if one allows the number $n$ of replica to be non-zero, the replica calculation (29), (33) gives the same expressions as for the random map models (40), (42) for $n=-1 / 2, \mu=0$ and (48), (49) for $n=-1, \mu=0$ or as the random walk problem (54), (55) for $n=-1, \mu=1 / 2$. Of course it would be very interesting to find other examples of systems with non-self-averaging effects which would correspond to the expressions obtained by the replica method (29), (33) for other values of $n$ and $\mu$. This would mean that the spin glass problem, at least in its mean field version, belongs to a larger class of problems [21], and it would then be very interesting to develop a more general theory than the presently existing replica approach to treat these more general problems.

The expressions obtained by the replica approach for a number of replica $n \neq 0$ has previously been considered in the literature. First, non-integer values of $n$ were considered to try to better understand the $n \rightarrow 0$ limit in the spin glass problem [22,17]. More recently, non-zero values of $n$ became important both as a mathematical tool to calculate the exact free energy of some disordered models [23], and also for physical reasons to describe situations where the quenched variables (the interactions $\left\{J_{i j}\right\}$ in the spin glass model) are no longer quenched but they are allowed to evolve according to slow dynamics [24].

As a final remark, I should say that the examples presented in this lecture could leave the impression that all problems with non-self-averaging effects are always special cases of (29) and (33) for some value of $n$ and $\mu$. This in fact is not true as one can easily build examples [25] for which the expressions of the moments of the $Y_{k}$ do not correspond to any value of $n$ and $\mu$.

\section{Further developments}

One can repeat the calculation of Section 2 on the sums of independent random variables in the case where the number $N$ of terms in the sum (2) is arbitrary but with the sum $S_{N}$ fixed at a certain (very large) value. One then finds [26] by an extension of the calculation of Section 2 that this case corresponds to the replica calculation (29), (33) with $n=-\mu$ and $-1<n<0$.

One can also show [26] that the general case (arbitrary $n$ and $\mu$ ) of the statistics predicted by the replica calculation can be achieved by the following construction of the weights $W_{\alpha}$ : define a sequence of random numbers $z_{1}, z_{2}, z_{3}, \ldots$ where each $z_{k}$ is distributed according to a distribution $\rho_{k}(z)$

$$
\rho_{k}(z)=\frac{\Gamma((k-1) \mu-n+1)}{\Gamma(1-\mu) \Gamma(k \mu-n)} z^{-\mu}(1-z)^{k \mu-n-1}
$$

and then construct the weights $W_{\alpha}$ by

$$
W_{1}=z_{1} \quad W_{2}=z_{2}\left(1-W_{1}\right) \quad W_{3}=z_{3}\left(1-W_{1}-W_{2}\right) \text { etc. }
$$

The $Y_{k}$ calculated from these random weights as in (1) have, for all choices of $n$ and $\mu$, the statistical properties predicted by the replica approach (29), (33). 
Closely related questions as the joint distribution of the $W_{\alpha}$ have been studied in the mathematical literature [27-31]. There also, there is a two parameter family of distributions (the Poisson Dirichlet distributions) which are, up to a change of notations, identical to the distributions discussed here (and indexed here $i$ th the two parameters $n$ and $\mu$ ). Also, as noted by Higgs [32], the statistical properties of the $Y_{k}$ play an important role in genetics.

We have seen in Section 4 that one step of broken replica symmetry gives the same statistics for the weights $W_{\alpha}$ and for the $Y_{k}$ as sums of independent random numbers. If one considers the broken replica symmetry with more than one step [33], as for example in the GREM $\left[8,34\right.$ ], the weights $W_{\alpha}$ can be regrouped into clusters and clusters of clusters, with more general correlations which can be calculated [33,34].

This text is an updated and extended version of [35].

\section{Acknowledgements}

I would like to thank $\mathrm{H}$. Flyvbjerg with whom the main ideas presented here have been developed. Also, I am very grateful to $\mathrm{A}$. Vershik and $\mathrm{M}$. Yor for very interesting discussions.

\section{References}

[1] M. Mezard, G. Parisi, N. Sourlas, G. Toulouse and M. Virasoro, Replica symmetry breaking and the nature of the spin glass phase, J. Physique 45 (1984) 843-854.

[2] M. Mezard, G. Parisi and M. Virasoro, Spin Glass Theory and Beyond (World Scientific, Singapore, 1987).

[3] J.P. Bouchaud and A. Georges, Anomalous diffusion in disordered media: Statistical mechanisms, models and physical applications, Phys. Rep. 195 (1990) 127-293.

[4] G. Parisi, The order parameter for spin glasses: A function on the interval 0-1, J. Phys. A 48 (1980) 1101-1112.

[5] D. Sherrington and S. Kirkpatrick, Solvable model of a spin glass, Phys. Rev. Lett. 35 (1975) 1792-1796.

[6] S. Kirkpatrick and D. Sherrington, Infinite ranged models of spin glasses, Phys. Rev. B 17 (1978) 4385-4403.

[7] B. Derrida, Random energy model, an exactly solvable model of disordered systems, Phys. Rev. B 24 (1981) $2613-2626$.

[8] B. Derrida and E. Gardner, Magnetic properties and the function $q(x)$ of the generalised random energy model, J. Phys. C 19 (1986) 5783-5798.

[9] B. Derrida, Directed polymers in a random medium, Physica 163 (1980) 71-84.

[10] J.L. van Hemmen and R.G. Palmer, The replica method and a solvable spin glass model, J. Phys. A 12 (1979) 563-580.

[11] D. Sherrington, Ising replica magnet, J. Phys. A 13 (1980) 637-649.

[12] A. Blandin, Theories versus experiments in the spin glass systems, J. Physique C 6 (1978) 1499-1516.

[13] J.R.L. de Almeida and D.J. Thouless, Stability of the Sherrington-Kirkpatrick solution of a spin glass model, J. Phys. A 11 (1978) 983-990.

[14] G. Parisi, Order parameter for spin glasses, Phys. Rev. Lett. 50 (1983) 1946-1948.

[15] B. Derrida and G. Toulouse, Sample to sample fluctuations in the random energy model, J. Physique Lett. 46 (1985) L223-L228.

[16] M. Mezard, G. Parisi and M.A. Virasoro, Random free energies in spin glasses, J. Physique Lett. 46 (1985) L217-L222.

[17] E. Gardner and B. Derrida, The probability distribution of the partition function of the random energy model, J. Phys. A 22 (1989) $1975-1981$.

[18] S.A. Kauffman, The Origin of Order (Oxford University Press, Oxford, 1993).

[19] B. Derrida and H. Flyvbjerg, Multivalley structure in Kauffman's model: Analogy with spin glasses, J. Phys. A 19 (1986) L1003L1008.

[20] B. Derrida and H. Flyvbjerg, The random map model, a disordered model with deterministic dynamics, J. Physique 48 (1987) 971-978.

[21] B. Derrida and H. Flyvbjerg, Statistical properties of randomly broken objects and of multivalley structures in disordered systems, J. Phys. A 20 (1987) 5273-5288.

[22] I. Kondor, Parisi's mean field solution for spin glasses as an analytic continuation in the replica number, J. Phys. A 16 (1993) L127-L131.

[23] E. Buffet, A. Patrick and J.V. Pule, Directed polymers on a tree: A martingale approach, J. Phys. A 26 (1983) $1823-1834$.

[24] R.W. Penney, A.C. Coolen and D. Sherrington, Coupled dynamics of fast spins and slow interactions in neural networks and spin systems, J. Phys. A 26 (1993) 3681-3695.

[25] B. Derrida and D. Bessis, Statistical properties of valleys in the annealed random map model, J. Phys. A 21 (1988) L509-L515. 
[26] B. Derrida and H. Flyvbjerg, unpublished.

[27] J. Pitman and M. Yor, Two studies of Poisson-Dirichlet distributions preprint 1995, to appear.

[28] J. Pitman and M. Yor, Random discrete distributions derived from self-similar random sets preprint 1995, to appear.

[29] A.M. Vershik, The asymptotic distribution of factorizations of natural numbers into prime divisors, Soviet Math. Dokl. 34 (1986) $57-61$.

[30] A.M. Vershik and A.A. Shmidt, Limit measures arising in the theory of groups I, Theor. Prob. Appl. 22 (1977) 79-85.

[31] A.M. Vershik and A.A. Shmidt, Limit measures arising in the theory of groups II, Theor. Prob. Appl. 23 (1978) 36-49.

[32] P.G. Higgs, Frequency distributions in population genetics parallel those in statistical physics, Phys. Rev. E 51 (1995) 95-101.

[33] G. Parisi, On the branching structure of the tree of states in spin glasses J. Stat. Phys. 72 (1993) 857-878.

[34] D. Ruelle, A mathematical reformulation of Derrida's REM and GREM, Comm. Math. Phys. 108 (1987) $225-239$.

[35] B. Derrida, Non-self-averaging effects in sums of random variables, spin glasses, random maps and random walks, in: On Three Levels: Micro, Meso and Macroscopic Approaches in Physics, eds. M. Fannes et al. (Plenum Press, New York, 1994) pp. $125-137$. 